NOTICE: this is the author's version of a work that was accepted for publication in the journal Measurement. Changes resulting from the publishing process, such as peer review, editing, corrections, structural formatting, and other quality control mechanisms may not be reflected in this document. Changes may have been made to this work since it was submitted for publication. A definitive version was subsequently published in the Journal Measurement, Vol.46, No.2 (2013). DOI: 10.1016/j.measurement.2012.10.015 


\title{
Performance Evaluation of Different Troposphere Delay Models and Mapping Functions
}

\author{
A. Tuka and A. El-Mowafy* \\ *Curtin University, Perth, Australia \\ Department of Spatial Sciences, Curtin University, Australia \\ GPO Box U 1987, Perth WA 6845, \\ A.el-mowafy@curtin.edu.au
}

\begin{abstract}
Tropospheric delay is a major error source in positioning by Global Navigation Satellite Systems (GNSS). System users often apply one of the available troposphere models without giving sufficient background on their performance. In this study, the performance of different known hydrostatic and wet troposphere delay models and mapping functions are internally and externally compared and analysed at selected sites around the globe. International GNSS Service (IGS) products were used as a reference. The best performing models are presented. Results showed that small discrepancies are present between different models. All models perform significantly better at the mid-latitudes than at the Equator.
\end{abstract}

\section{Keywords:}

Troposphere delay, GNSS, mapping functions, wet delay, hydrostatic delay

\section{Introduction}

In GNSS positioning, troposphere delay error typically range between $2.0 \mathrm{~m}$ and $2.5 \mathrm{~m}$. This delay comprises two components; a hydrostatic part, which constitutes more than $90 \%$ of the total troposphere delay, and a wet part, which is usually less than $10 \%$ of this delay [14]. Traditionally, the hydrostatic component can be estimated to an accuracy of better than $90 \%$ using empirical models that utilizes metrological data, such as pressure and temperature, as well as the position of the user. Although the wet troposphere is difficult to model due to the variability of the water vapour in the upper atmospheric layer, some models were developed to estimate the wet troposphere delay if no such information is available.

In practice, user often employs a certain troposphere model based on popularity of the model without giving enough justification. Limited comparisons between some of the models have been carried out in the past for local or regional applications [e.g. 11]. However, in this contribution, this issue is addressed more comprehensively where the performance of various models used in estimating the hydrostatic and wet tropospheric delays is compared and analysed. The zenith hydrostatic delay (ZHD) models analysed are the Saastamoinen, Hopfield, Baby, Davis, and Askne and Nordius. The zenith wet delay (ZWD) models considered are the Saastamoinen, Hopfield, Baby, Ifadis, Askne \& Nordius, and Berman. In addition, due to the fact that the troposphere delay is typically estimated along the zenith direction and projected along the user-to-satellite line of sight, different mapping functions were developed for this purpose. Thus, it is of interest when taking into account the effect of 
troposphere delay is to address performance of different mapping functions that can be used. Therefore, the study also includes comparing performance of different creditable mapping functions. The mapping functions that are examined are the Davis, Ifadis, Chao, Herring, Niell, Global and Vienna mapping functions.

The above troposphere models however may perform differently in accordance with the location of the user [13]. Thus, one objective of this paper is to investigate the performance of these models at selected sites across the globe of interest to Australian users, which include Antarctica, Australia, the Equator, England, and USA. Testing is performed using demonstrative data that span two months in the summer and two months in the winter (January - February, and July-August, 2010). Although the use of a larger pool of points and over longer periods is desirable, this study would give a good indication about what one would expect from the tested models at similar conditions.

\section{Troposphere modelling in the GNSS observation equations}

The troposphere delay $T^{k}$ of GNSS signal propagation between receiver $i$ and satellite $k$ can be given as follows [20]:

$$
T_{i}^{k}=\int_{i}^{k}(n-1) d s
$$

where $n$ is the refractive index of the medium the signal is passing through, defined as the ratio of the speed of propagation of an electromagnetic wave in a vacuum to the speed of propagation in this medium, and $d s$ is a small distance along the signal path. The GNSS code and phase observation equations can be formulated as [14]:

$$
\begin{aligned}
& P_{(t)}=R_{i(t, t-\tau)}^{k}+d \rho_{(t-\tau)}^{k}+c d t_{i(t)}-c d t_{(t-\tau)}^{k}+I^{k}{ }_{i+} T^{k}{ }_{i}+d p^{k}{ }_{i}+I F+\varepsilon_{i}^{k}(\rho) \\
& \phi_{(t)}=R_{i(t, t-\tau)}^{k}+d \rho_{(t-\tau)}^{k}+c d t_{i(t)}-c d t_{(t-\tau)}^{k}-I^{k}{ }_{i+} T^{k}{ }_{i}+d \phi^{k}{ }_{i}+N^{k}{ }_{i}+\varepsilon_{i}^{k}(\phi)
\end{aligned}
$$

where $P_{(t)}$ and $\phi_{(t)}$ are the code and the phase measurements received at time $t$, respectively. $R_{i}^{k}$ is the receiver-to-satellite range, $d \rho^{k}$ is the orbital error, $\tau$ is the time taken by the signal to travel from the satellite to the receiver, $c$ denotes the speed of light, $d t_{i}$ and $d t^{k}$ are the receiver $i$ and satellite $k$ clock errors. $I_{i}^{k}$ is the ionosphere error, and $T^{k}{ }_{i}$ denotes the total troposphere delay. $d p^{k}{ }_{i}$ is the receiver and satellite hardware code biases and $d \phi_{i}^{k}$ includes the receiver and satellite hardware phase biases and the initial phase biases. Both terms also include smaller errors such as the relativistic error, Sagnac delay, receiver and satellite antenna-phase centre offsets and variations, site displacement effects due to Earth tide, ocean tide and atmospheric loading [14]. IF denotes the inter-frequency bias, and $N^{k}{ }_{i}$ is the integer

phase ambiguity. Finally, $\varepsilon_{i}^{k}(\rho)$ and $\varepsilon_{i}^{k}(\phi)$ are the code and phase noises, which are usually assumed Gaussian with zero mean. Most of these errors are minimised by differencing over short to medium distances, and in case of the ionosphere, its first order term can be eliminated by the use of dual-frequency ionosphere-free linear combination of the observations.

To avoid the problem of having under-determined system when solving the above equations, due to the need to estimate the slant troposphere error for each satellite, the troposphere delay is generally expressed at each ground location in terms of one value taken along the zenith, i.e. Zenith Total Delay (ZTD). This one value of ZTD is used for all satellites 
observed from a single location where a mapping function is utilised to project the ZTD onto the receiver-to-satellite line of sight direction for each satellite, such that [19]:

$$
T_{i}^{k}=m\left(\theta_{i}^{k}\right) * Z T D_{i}
$$

where $\theta_{i}^{k}$ is the elevation angle between the receiver $i$ and the satellite $k, m\left(\theta_{i}^{k}\right)$ is the mapping function and $Z T D_{i}$ is the $Z T D$ at receiver $i$. Traditionally, the hydrostatic and wet components of the total troposphere delay are treated separately. In this case, two mapping functions are needed for the hydrostatic and wet delays, denoted as $m_{H}$ and $m_{W}$. The total troposphere delay can then be expressed as [19]:

$$
T_{i}^{k}=m_{H}\left(\theta_{i}^{k}\right) Z H D_{i}+m_{W}\left(\theta_{i}^{k}\right) Z W D_{i}
$$

where $Z H D_{i}$ and $Z W D_{i}$ are the Zenith hydrostatic and wet components of the total troposphere delay at station $i$. For the traditional double-difference observations between the stations $i, j$ and the satellites $k, l$, the troposphere delay term can be formulated as:

$$
\begin{aligned}
T_{i j}^{k l}= & Z T D_{i}\left(m_{i}^{k}-m_{i}^{l}\right)-Z T D_{j}\left(m_{j}^{k}-m_{j}^{l}\right) \\
& =Z T D_{i} * \Delta m_{i}^{k l}-Z T D_{j} * \Delta m_{j}^{k l}
\end{aligned}
$$

where $\Delta m$ denotes the differential mapping functions computed for the two satellites under consideration. In this paper, the results from different empirical models used for estimation of the ZHD and ZWD, and mapping functions $m_{H}$ and $m_{W}$ are compared and analysed.

\section{Model parameters}

Due to the large number of tested hydrostatic and wet zenith delay models and limitations of the paper length, interested reader can find details of these models including their theory, principles, assumptions and equations in the list of references, given for each model in the first column in Table 1. The table also summarises the input parameters for the analysed models. These parameters are given to show which Meta data each model is using, where: $e$ is the surface water vapour pressure; $T$ denotes the surface temperature; $P$ is the surface pressure; $\beta$ is the temperature lapse rate; $\varphi$ and $\lambda$ denote station latitude and longitude; $H$ is height of station; $g_{s}$ is the surface gravity; $R_{d}$ is specific gas constant for dry air; $r$ is the Earth's radius; $U$ denotes relative humidity; $\Lambda, v, k_{1}, k_{2}, k_{3}, k_{2}^{\prime}$ and $\gamma$ are empirical coefficients. One can see that all hydrostatic models (except Hopfield model) require knowledge of the user latitude, height and surface pressure, whereas most wet delay models require knowledge of the surface temperature as well as the surface water vapour pressure.

Table 2 shows the coefficients and input parameters for the mapping functions under analysis, denoted as MFs. Their details can be found in the references given in the first column of the table. The input parameters are defined as above, in addition to $h_{t}$ which denotes height of the tropopause, $D O Y$ is the Coordinated Universal Time (UTC) day since the beginning of the year, $d_{m j d}$ denotes modified Julian date, $\theta$ is again the elevation angle, $a_{h}$ and $a_{w}$ are coefficients for determining Vienna Mapping Functions (VMF1). The last 
column in Table 2 gives the type of MF in each model, where $m_{H}$ and $m_{W}$ denote the hydrostatic and wet mapping functions, respectively.

Table 1: Parameters of different troposphere delay models

\begin{tabular}{|c|c|c|}
\hline \multirow{2}{*}{$\begin{array}{l}\text { Tropospheric } \\
\text { Delay Models }\end{array}$} & \multicolumn{2}{|c|}{ Parameterisation } \\
\cline { 2 - 3 } & Hydrostatic model & Wet model \\
\hline Hopfield [16] & $T, P, k_{1}$ & $T, e, k_{1}, k_{2}, k_{3}$ \\
\hline Saastamoinen [25] & $\varphi, P, H$ & $T, e$ \\
\hline Berman [3] & ----- & $T, e, \beta$ \\
\hline Davis [10] & $\varphi, P, H$ & ------ \\
\hline Ifadis [17] & ----- & $T, e, P$ \\
\hline Askne [1] & $\varphi, T, P, k_{1}, R_{d}, H$ & $\begin{array}{c}\varphi, T, e, H, \beta, k_{1} \text { and } \\
k_{2}, k_{3}, k_{2}^{\prime}, R_{d}, \Lambda\end{array}$ \\
\hline Baby [2] & $T, P, \beta, g_{s}, r, H, R_{d}$ & $T, U, v, y$ \\
\hline
\end{tabular}

Table 2: Input parameters of different troposphere mapping functions

\begin{tabular}{|l|c|c|}
\hline \multirow{2}{*}{\multicolumn{1}{c|}{$\begin{array}{c}\text { Mapping } \\
\text { Function }\end{array}$}} & \multicolumn{2}{c|}{ Parameterisation } \\
\cline { 2 - 3 } & INPUT & MF \\
\hline Chao [9] & $\theta$ & $\mathrm{m}_{\mathrm{H}}, \mathrm{m}_{\mathrm{W}}$ \\
\hline CfA2.2 [10] & $\theta, e, P, T, b, h_{t}$ & $\mathrm{~m}_{\mathrm{H}}$ \\
\hline Ifadis [17] & $\theta, e, P, T$ & $\mathrm{~m}_{\mathrm{H}}, \mathrm{m}_{\mathrm{W}}$ \\
\hline Herring [15] & $\theta, T, H, \varphi$ & $\mathrm{m}_{\mathrm{H}}, \mathrm{m}_{\mathrm{W}}$ \\
\hline NMF [23, 24] & $\theta, D O Y, H, \varphi$ & $\mathrm{m}_{\mathrm{H}}, \mathrm{m}_{\mathrm{W}}$ \\
\hline VMF1 [5, 7] & $\theta, d_{m j d}, H, \varphi, a_{h}, a_{w}$ & $\mathrm{~m}_{\mathrm{H}}, \mathrm{m}_{\mathrm{W}}$ \\
\hline GMF [6] & $\begin{array}{c}\theta, d_{m j d} H, \varphi, \lambda \\
\text { and ERA40 data at } \\
15^{\circ} \times 15^{\circ}\end{array}$ & $\mathrm{m}_{\mathrm{H}}, \mathrm{m}_{\mathrm{W}}$ \\
& \multicolumn{2}{|c|}{} \\
\hline
\end{tabular}

\section{Test description}

In order to compare the various tropospheric delay models and mapping functions considered in this study, the ZTD product from the IGS was used as a reference. The IGS ZTD are computed by using the GIPSY software in a precise point positioning (PPP) mode. Niell mapping functions and hydrostatic delay modelling were used, whereas the wet zenith delay is estimated [8, 12]. The IGS products are determined using GNSS data from the Global Positioning System (GPS) with a 30 second sampling rate. Although it would be best to include all worldwide IGS stations in this study, and to apply them over a very long period of time, however, for demonstration purposes the tested models were compared using four months of continuous data in 2010. The first two months were January and February 2010 (to represent summer in the southern Hemisphere and winter in the northern 
Hemisphere). The second set comprises two months of continuous data (July and August, 2010), which represent winter in the southern Hemisphere and summer in the northern Hemisphere. This session selection of data over a relatively long period would give well represented sample of data over different weather conditions. Six IGS stations were chosen for testing at different regions across the globe, including three stations from the Southern Hemisphere, one on the equator, and two from the Northern Hemisphere. The three stations from Southern Hemisphere are PERT (Perth, Western Australia), HOB2 (Hobart, Tasmania) and DAV1 (Davis site, Antarctica). The station used on the Equator was DGAR (Diego Garcia Island). The stations used in the Northern Hemisphere were MDO1 (Texas, USA) and HERT (Hailsham, England). Figure 1 shows the locations of test points. Their ellipsoidal heights in the World Geodetic System-84 (WGS84) are given in Table 3.

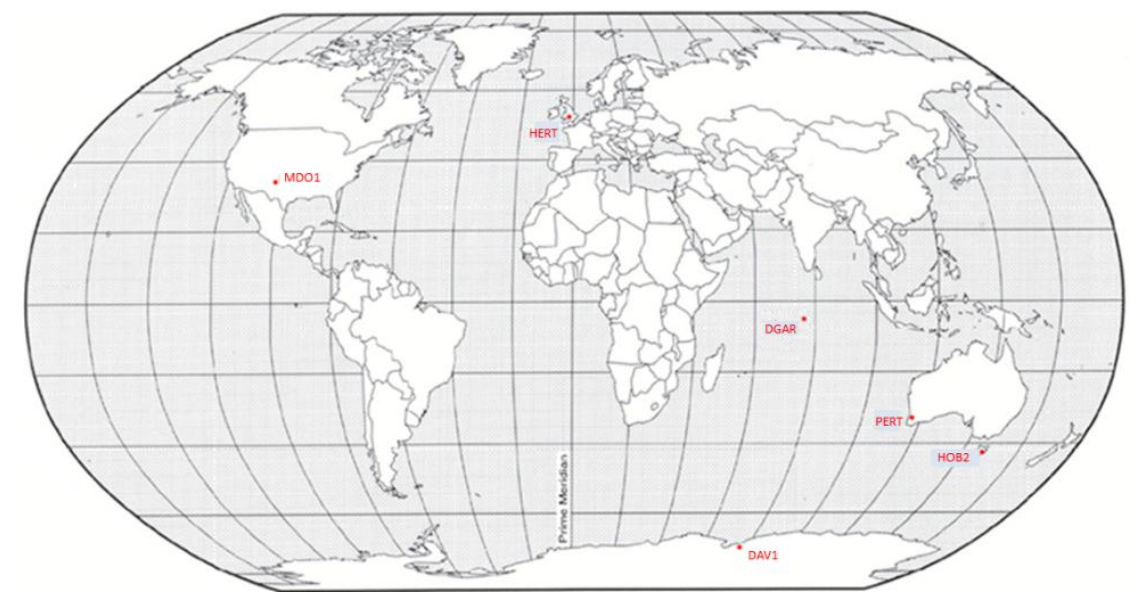

Figure 1: Location of IGS stations used in testing

Table 3: Ellipsoidal heights of the test points (m)

\begin{tabular}{|c|c|c|c|c|c|c|}
\hline Station & PERT & HOB2 & DAV1 & DGAR & MDO1 & HERT \\
\hline Ellipsoidal height & 12.920 & 41.127 & 44.500 & -64.746 & 2004.476 & 83.300 \\
\hline
\end{tabular}

For each particular station, meteorological data were needed for use in some of the tested models. Thus, data from the nearest available sites to the test IGS stations were used. For the HOB2 and PERT stations, meteorological data were obtained from the Australian Bureau of Meteorology. For the Davis station, meteorological data were obtained from the Australian Antarctic Division. For the Equator and USA stations, meteorological data were obtained from the National Oceanic and Atmospheric Administration, and lastly the meteorological data for station HERT in England was obtained from a weather website.

\section{Comparison of ZHD models}

In view of the fact that no accurate external source was available for comparing the ZHD values, only internal comparison between the different models was carried out choosing the Saastamoinen model as a reference, and the differences between the considered ZHD models and the Saastamoinen model were determined. This choice was based on previous testing, which showed that Saastamoinen model results agreed with ray tracing results at the submillimetre level $[18,21,22]$. The models compared to the Saastamoinen model were Hopfield, Baby, Davis, and Askne and Nordius. ZHD values were computed from each of these models using the test data of four months with a sampling interval of 30 seconds and 
the mean value of all differences between the computed ZHD from these models and Saastamoinen model are shown in Figure 2 for all six test sites.

The top plot in the Figure 2 shows results from the Northern Hemisphere stations MDO1 (USA) and HERT (England) and the Equator station (DGAR) combined. The bottom part of the figure shows results from the three southern Hemisphere stations PERT, HOB2 and DAV1. The left figures show the data of January and February and the right figures show the data of July and August, 2010. The figure shows that the largest difference from Saastamoinen is for the Hopfield model for the station MDO1. A factor contributing to this difference is that the station MDO1 is significantly higher than the stations HERT and DGAR by approximately $1921 \mathrm{~m}$ and $2068 \mathrm{~m}$, respectively. This may indicate that the Hopfield model is affected by ignoring point heights. It was closer to Saastamoinen at higher elevations than at low elevations. Figure 2 also shows that the smallest deviations occur near the Equator, and the largest (more than $9 \mathrm{~mm}$ ) are at the Antarctica station for the Hopfield model. This effect can be explained by missing the gravity reduction in the Hopfield approach which treats the gravity as a constant value [16]. The Baby model expresses almost a constant difference from Saastamoinen by about $-5 \mathrm{~mm}$ for each IGS station. This is due to the fact that the equation used by Baby to estimate the gravity is a function of surface temperature and temperature lapse rate. The use of a global value of $6.5 \mathrm{~K} / \mathrm{km}$ for the temperature lapse rate reduces the performance of the model. The other two models, Davis, and Askne and Nordius gave results very close to Saastamoinen, as their principles are based on the Saastamoinen model, but use different values for determining their coefficients.
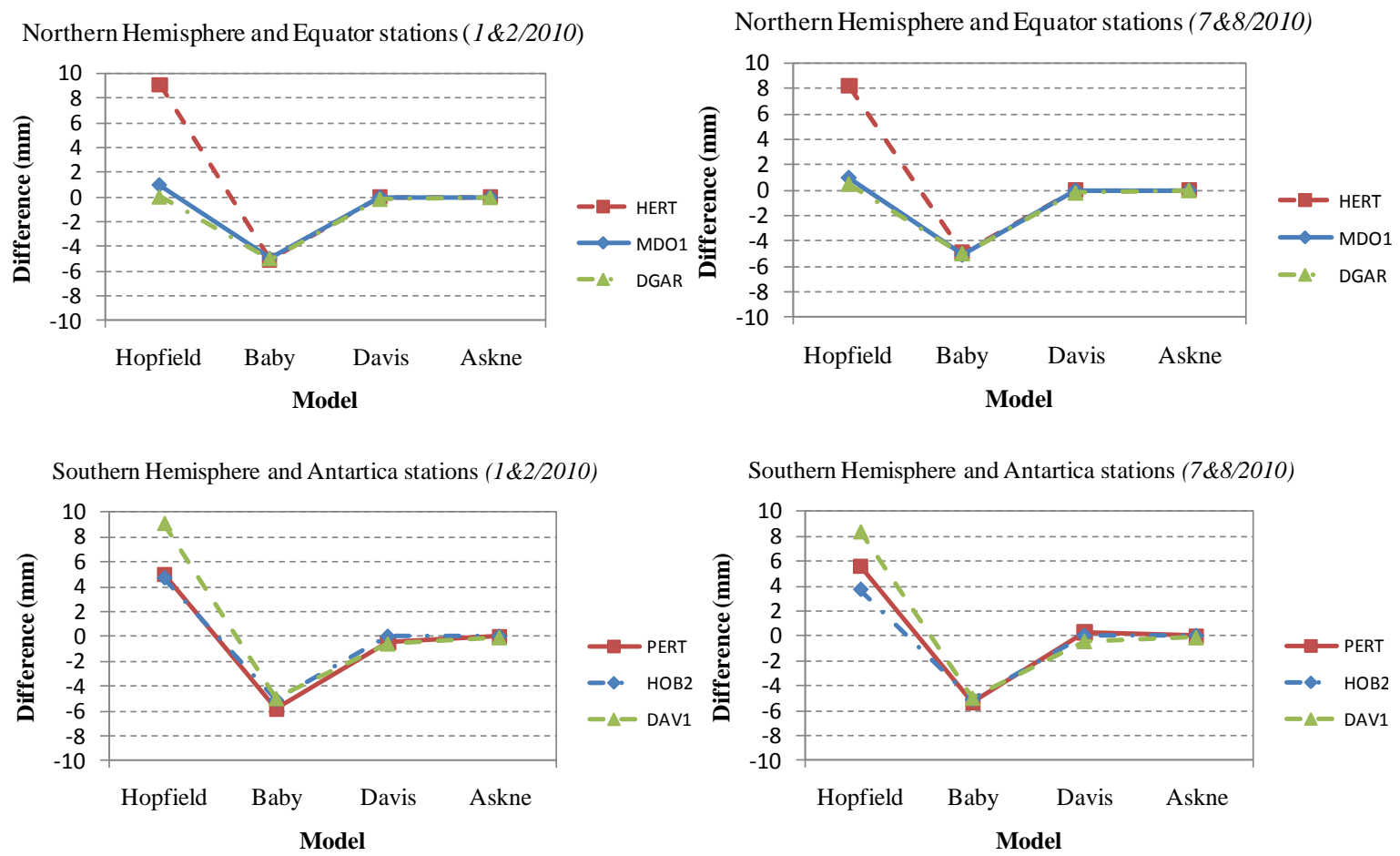

Figure 2: $\quad$ Differences between ZHD models referenced to Saastamoinen model

Figure 2 shows that the differences between the compared models follow almost a similar pattern for both test periods January - February and July - August, indicating that similar results could be obtained all around the year. Descriptive statistics of the differences between results of different models and Saastamoinen model are given in Table 4. Overall, the Hopfield model tends to overestimate the zenith delay, except for the Equator and USA 
IGS stations. The Baby model tends to underestimate the zenith delay compared with Saastamoinen. The Davis, and Askne and Nordius models perform evenly with Saastamoinen model, as they gave the exact values for the ZHD. Results show that differences can be considered insignificant for a wide range of applications, in particular when a differenced processing technique is applied between pairs of stations or in a network processing over short to medium distances.

Table 4: Differences among ZHD models and Saastamoinen model (mm)

\begin{tabular}{|c|c|c|c|c|}
\hline Model & Mean & Range & $\begin{array}{c}\text { standard deviation } \\
(\boldsymbol{\sigma})\end{array}$ & $\begin{array}{c}\text { difference } \\
\text { from mean } \\
\%\end{array}$ \\
\hline Hopfield & 3.43 & 9.31 & 2.52 & 0.16 \\
\hline Baby & -5.11 & 1.62 & 0.41 & -0.23 \\
\hline Davis & -0.18 & 0.05 & 0.02 & -0.01 \\
\hline $\begin{array}{c}\text { Askne \& } \\
\text { Nordius }\end{array}$ & -0.20 & 0.28 & 0.10 & -0.01 \\
\hline
\end{tabular}

\section{Comparison of ZWD models}

The difficulty of modelling the water vapour pressure, and accordingly the wet troposphere delay, due to its high irregularity can be shown, in some sense, when comparing different empirical models used for estimating ZWD. The estimated ZWD values at the six test stations ranged from $11 \mathrm{~mm}$ to $93 \mathrm{~mm}$. Once more, the Saastamoinen model was used as a reference for the internal comparison. The ZWD models compared to the Saastamoinen model were Hopfield, Baby, Ifadis, Askne and Nordius, and Berman. The mean values of all differences between ZWD values estimated from each of these models and ZWD values determined from Saastamoinen are shown in Figure 3 for the January-February period, shown in the left part of the Figure, and July-August, illustrated on the right part of the figure. In Figure 3, the top plot represents the Northern Hemisphere and Equator stations MDO1, HERT and DGAR combined. Results of MDO1 and HERT show that the ZWD model differences from Saastamoinen range from $-13 \mathrm{~mm}$ to $21 \mathrm{~mm}$. There was also a significant variation in the Ifadis and Berman model when comparing results at the two stations. For DGAR station, situated at the Equator, the differences were significant, were they were $-32 \mathrm{~mm}$ on average for Baby, and $50 \mathrm{~mm}$ using Berman. The bottom part of the Figure 3 illustrates the results of the southern Hemisphere stations PERT, HOB2 and DVA1. For Pert, Babay and Berman again gave the largest discrepancies at $-22 \mathrm{~mm}$ and $38 \mathrm{~mm}$, respectively. For Antarctica station DAV1, the model differences ranged from $-2 \mathrm{~mm}$ to 11 $\mathrm{mm}$.
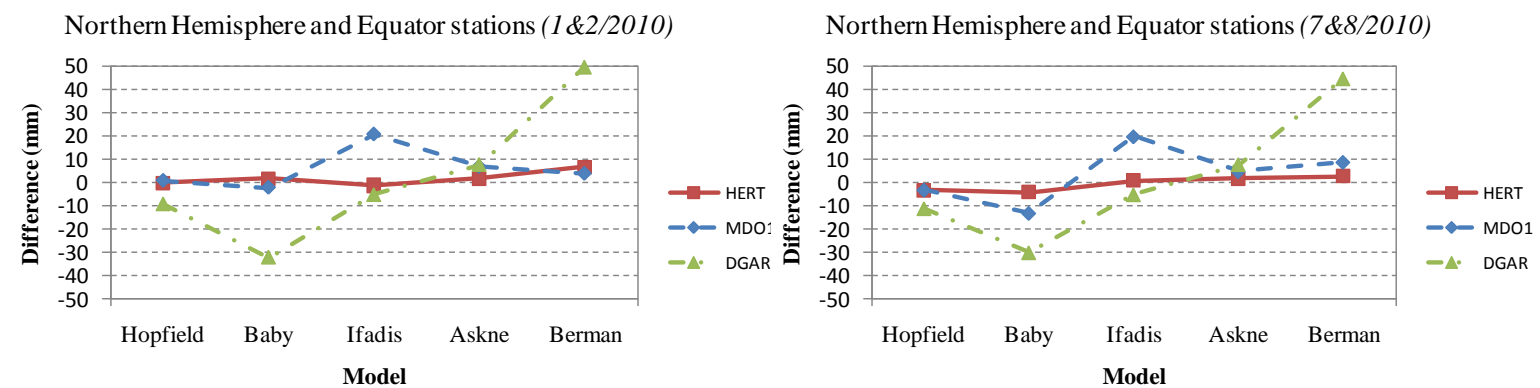

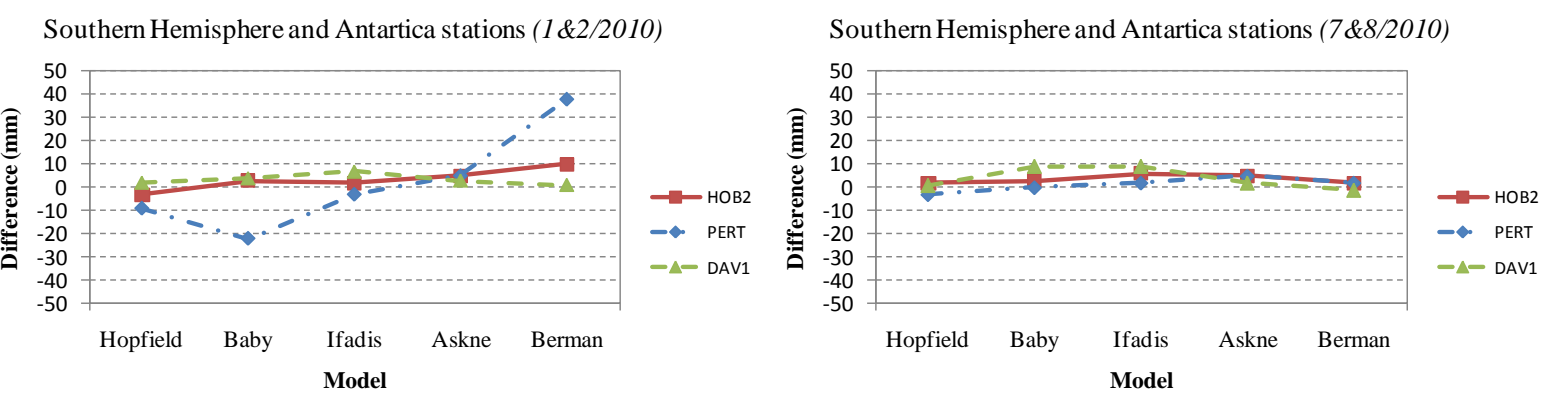

Figure 3: $\quad$ Differences between ZWD models referenced to Saastamoinen

From these results, one can see that the ZWD models performed poorly at the Equatorial station and best at the Antarctica station, due to the fact that the Equatorial region experiences higher amounts of water vapour pressure in the troposphere than at the Antarctica station. The worst performing model was the Berman. The reason why the Berman model performed poorly was that this model is based on fixed laboratory coefficients that do not apply to any region that has been focused on in this study except for the Antarctica station. The Baby model performs badly at some sites due to the fact that this model is affected significantly by the variation of relative humidity at each site. Since the nearest meteorological data was used, not the actual site meteorological data, the Baby model is adversely affected.

Comparison of different model results shown in Figure 3 illustrates that ZWD estimation cannot be easily estimated as the ZHD from the given models for the global figure, mainly due to the high variation in the water vapour profiles at different sites. Although Figure 3 shows that all models almost follow a similar pattern; the differences between the tested models and Saastamoinen model were on average about $58 \%$ of the ZWD values. One or two parameters used in these models are often insufficient to describe the variations of the humidity with height. For some instances, as for the case of Antarctica station DAV1, the model differences have exceeded the original ZWD values. The large differences seen explain why these models are not widely used.

\section{Comparison of ZTD models}

The two previous sections only demonstrate the internal comparisons among various ZWD and ZHD model results, using the Saastamoinen model as reference, due to the lack of accurate external ZHD and ZWD products for our study. However, in order to show how well each model represents the total troposphere delay, the IGS final ZTD product is used as an external reference, which has an accuracy of $4 \mathrm{~mm}$ and a sample interval of 2 hours. The ZTD is determined for the four models: Saastamoinen, Hopfield, Baby, and Askne and Nordius, which have ZHD and ZWD sub-models, by adding the ZWD to the ZHD. Other models such as the Ifadis and Berman only provide ZWD computation while the Davis model only provides ZHD computation, thus, they were not examined when testing the ZTD. Figure 4 illustrates the average differences between the tested four models and IGS ZTD results for the test period, where again the northern Hemisphere stations as well as the Equator station results are illustrated in the top part of the figure and the southern Hemisphere stations are illustrated in the bottom part of the figure. Table 5 gives the statistics of these differences.

Table 5: Differences between tested models and IGS ZTD (mm) 


\begin{tabular}{|c|c|c|c|c|}
\hline Model & Mean & Range & $\sigma$ & $\begin{array}{c}\text { Difference } \\
\text { from mean \% }\end{array}$ \\
\hline Saastamoinen & 3.57 & 62.56 & 16.30 & 0.16 \\
\hline Hopfield & 3.68 & 78.21 & 22.68 & 0.17 \\
\hline Baby & -8.90 & 87.44 & 31.55 & -0.59 \\
\hline $\begin{array}{ll}\text { Askne } & \& \\
\text { Nordius } & \end{array}$ & 7.36 & 46.23 & 15.23 & 0.33 \\
\hline
\end{tabular}

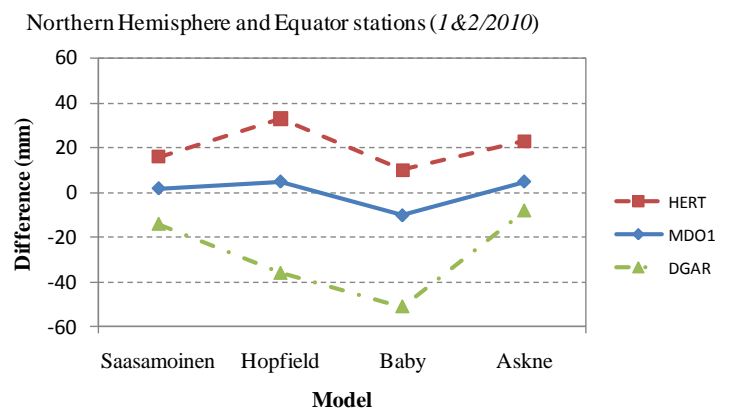

Southern Hemisphere and Antartica stations (1\&2/2010)

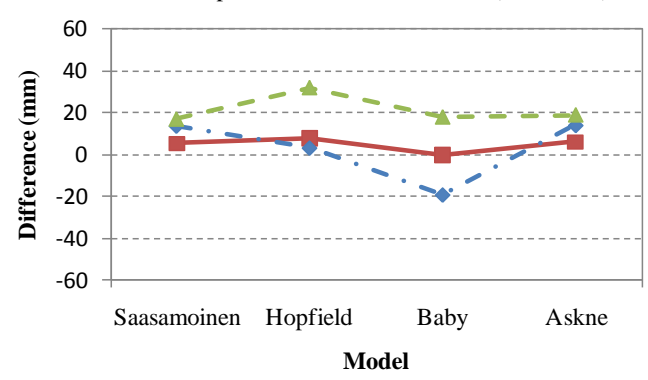

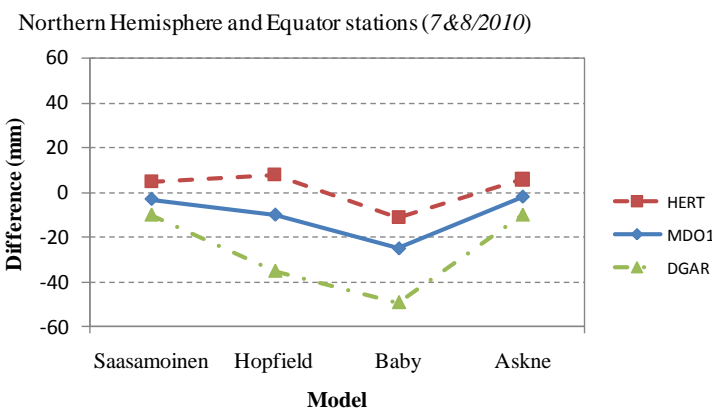

Southern Hemisphere and Antartica stations $(7 \& 8 / 2010)$

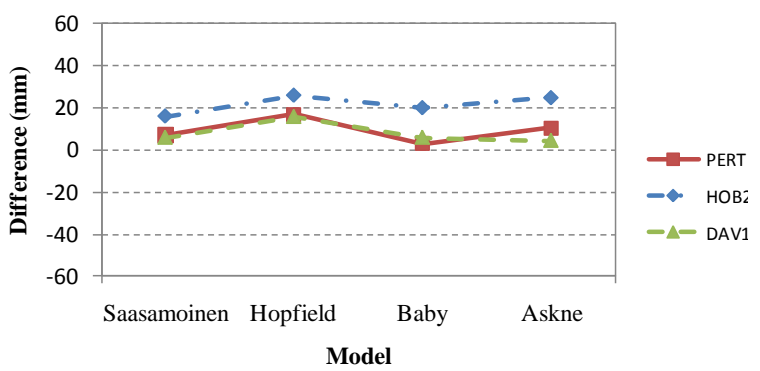

Figure 4: Differences between ZTD models, with respect to reference IGS ZTD

Results of the Northern Hemisphere stations MDO1 and HERT in Figure 4, show that Saastamoinen and Askne \& Nordius gave the best results compared with IGS products, whereas discrepancies between Hopfield from the reference IGS ranged from $9 \mathrm{~mm}$ to 33 $\mathrm{mm}$, and for Baby the discrepancies were from $-9 \mathrm{~mm}$ to $-23 \mathrm{~mm}$. These results were echoed when considering the Equator station DGAR, showing good agreement between Saastamoinen and Askne \& Nordius with IGS output, whereas for Hopfield and Baby even larger differences were observed at $-36 \mathrm{~mm}$ and $-51 \mathrm{~mm}$. The ZTD models differ most from the IGS ZTD product at the Equatorial station partly due to high content of water vapour pressure at the Equator which affects estimation of the ZTD.

Similar performance of the models was obtained for the southern Hemisphere stations, where model differences the differences were between $-19 \mathrm{~mm}$ and $34 \mathrm{~mm}$. The maximum differences were observed at the Antarctica station DAV1 in the summer at the level of 20 $\mathrm{mm}$ for all models except for Hopfield model, where the differences exceeded $30 \mathrm{~mm}$. The level of differences in the summer was generally lower than that in the winter due to the dryer weather. From the above results one can see that Saastamoinen and Askne and Nordius models give the best overall results for all examined IGS ZTD output stations combined and the Baby model performed the worst as it tends to overestimate the zenith delay. 


\section{Comparison of different mapping functions}

Mapping functions are used for the projection of the estimated tropospheric zenith delay to the slant tropospheric delay. In order to analyse the available mapping functions (MF), comparison is internally performed between the tested methods listed in Table 2 and using the VMF1 as a reference, which is currently providing globally the most accurate and reliable results with coefficients given at 6 hourly time intervals $[4,5,6,19,26]$.

For comparison purposes, and to show the impact of location on results, three test locations were chosen; one from the Southern Hemisphere (PERT station), one from the Equator (DGAR station), and another station from the Northern Hemisphere (MDO1). The differences between the tested MFs compared to the VMF1 are multiplied by the ZTD to present the differences in terms of the final tropospheric delay differences. These differences are split into the hydrostatic $\left(\Delta T_{H}\right)$ and the wet $\left(\Delta T_{W}\right)$ tropospheric delay differences, which read:

$$
\begin{gathered}
\Delta T_{H}=\Delta m_{H} \cdot Z H D \\
\Delta T_{W}=\Delta m_{W} \cdot Z W D
\end{gathered}
$$

where $\Delta m_{H}$ is the difference in the hydrostatic MF $\left(m_{H}\right)$ between the tested model and VMF1. Similarly, $\Delta m_{W}$ is the difference of the wet MF $\left(m_{W}\right)$ to the reference VMF1.

An example of changes of the MFs is illustrated in Figure 5. The figure depicts the VMF1 results for both the hydrostatic and wet MF for PERT station computed on $19^{\text {th }}$ of January 2010, which shows that the value of the MF increases as the elevation angle decreases. As a result, a satellite at elevation angle of $5^{\circ}$ will experience approximately 10 times tropospheric errors compared with a satellite observed at more than $35^{\circ}$ elevation angle. Both $\mathrm{m}_{\mathrm{H}}$ and $\mathrm{m}_{\mathrm{W}}$ follow a similar pattern; with a slight change at around $5^{\circ}$ elevation angle.

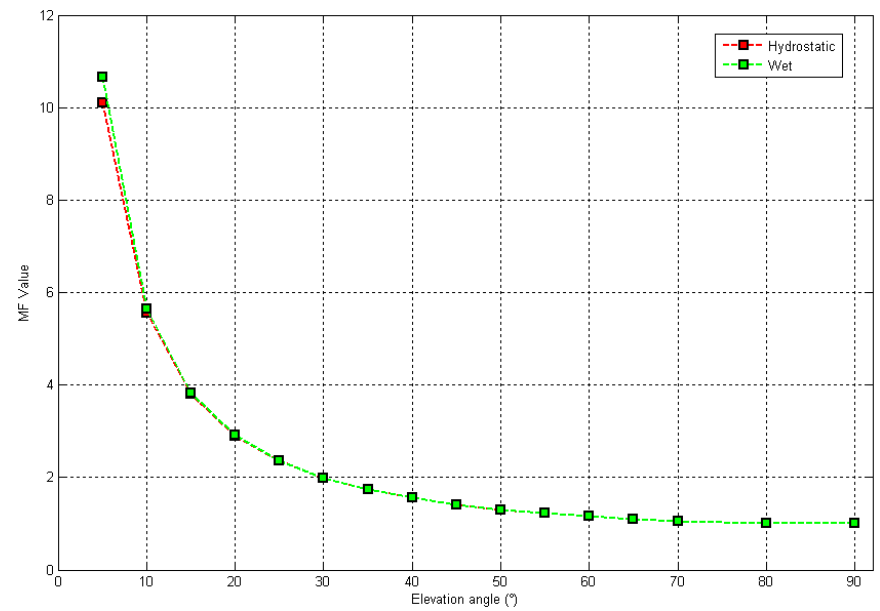

Figure 5: $\quad$ Values of Vienna $m_{H}$ and $m_{W}$ mapping functions at PERT

A comparison between different models and VMF1 at station PERT is shown in Figure 6. The differences are given as percentages and for the hydrostatic and wet models. The figure shows that the differences for all models increase as the elevation angle decreases. The worst 
performer of the $m_{H}$ was the Davis model, which reached a difference of $-13 \%$ from VMF1, followed by the Herring model which gave a difference of $-4.5 \%$, while the other hydrostatic MF model differences ranged from $-0.2 \%$ to $1.1 \%$. For the wet mapping functions, Chao and Herring performed the worst, with differences of $3.7 \%$ and $-3.1 \%$ respectively from VMF1. The Ifadis, Global and Niell MFw showed a similar pattern, with differences of $1 \%$ from VMF1. This could be due to the fact that the older models require quite accurate meteorological data for the stations, whereas the newer models do not need meteorological inputs. The use of numerical models of weather forecasts is becoming widely acceptable for this purpose.
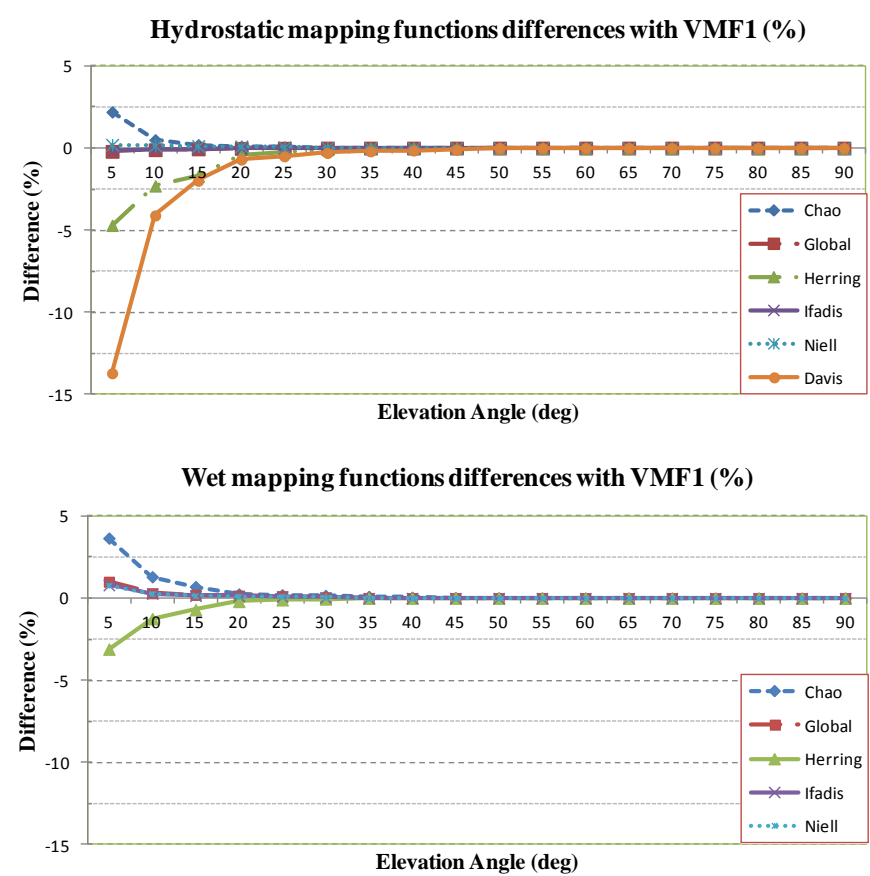

Figure 6: $\quad$ Percentage of differences between MFs and VMF1 at PERT

In order to quantify the impact of the differences between various MFs considered on the troposphere delay, the differences between the MFs and VMF1 were multiplied by the Saastamoinen ZHD and ZWD to represent the impact on the measured ranges in metre units. This is shown in Figure 7 for PERT station. At the lower elevation angles, the Davis model error reached $-3 \mathrm{~m}$. The Herring hydrostatic mapping function differed by -1 metre, while the other mapping function differences were within a range of $-0.1 \mathrm{~m}$ to $0.3 \mathrm{~m}$ from VMF1. For the wet mapping functions, the Herring and Chao MF gave difference of $-0.059 \mathrm{~m}$ and 0.069 $\mathrm{m}$, while the Ifadis, Global and Niell MFw showed a similar pattern, with mean differences of $0.02 \mathrm{~m}$ from VMF1. Similar plots for the test sites at the Equator and Northern Hemisphere sites (DGAR and MDO1) are given in the Figures 8 and 9. 

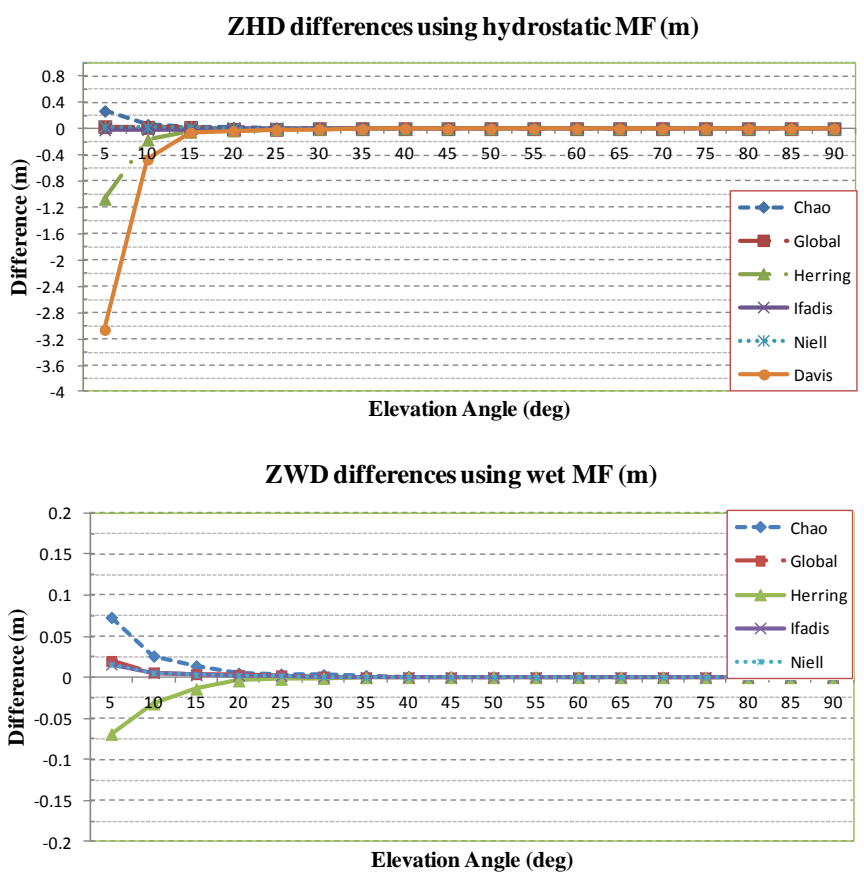

Figure 7: ZHD and ZWD differences between tested MF and VMF1 for PERT (m)

Comparing the results shown in the figures 7 and 9 show that the obtained ZHD and ZWD values applying the Niell MFs change sides when differenced from those of VMF1 as we move from the southern Hemisphere to the northern Hemisphere. This can be attributed to the fact that Niell assumed that the north and south latitudes are symmetric in calculating its coefficients. For the hydrostatic component, the Davis MFs gave the largest differences from VMF1 due to the fact that the calculation of the temperature lapse rate and tropopause height in the model is assumed related to the surface temperature. The hydrostatic Herring MFs performed badly as well. For the wet component, the Herring and Chao MFs performed worst at PERT and Equatorial station (DGAR).

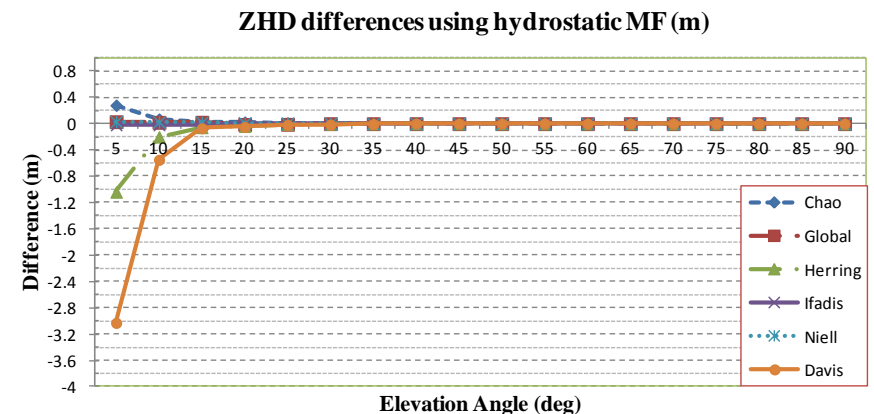




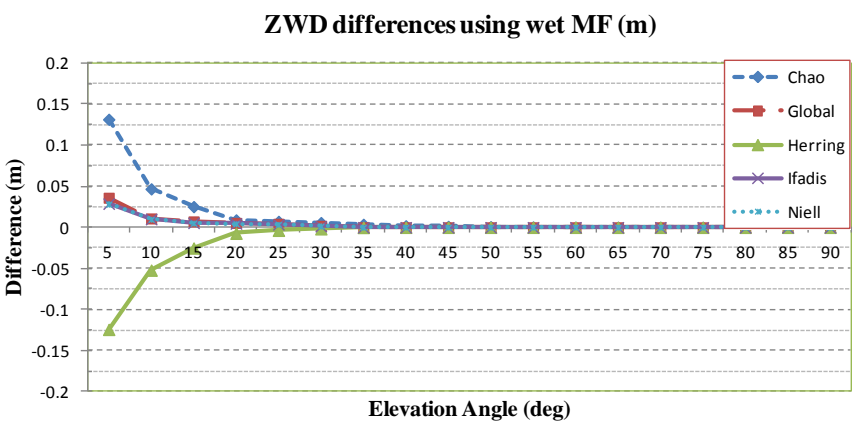

Figure 8: ZHD and ZWD differences between tested MF and VMF1 for DGAR (m)
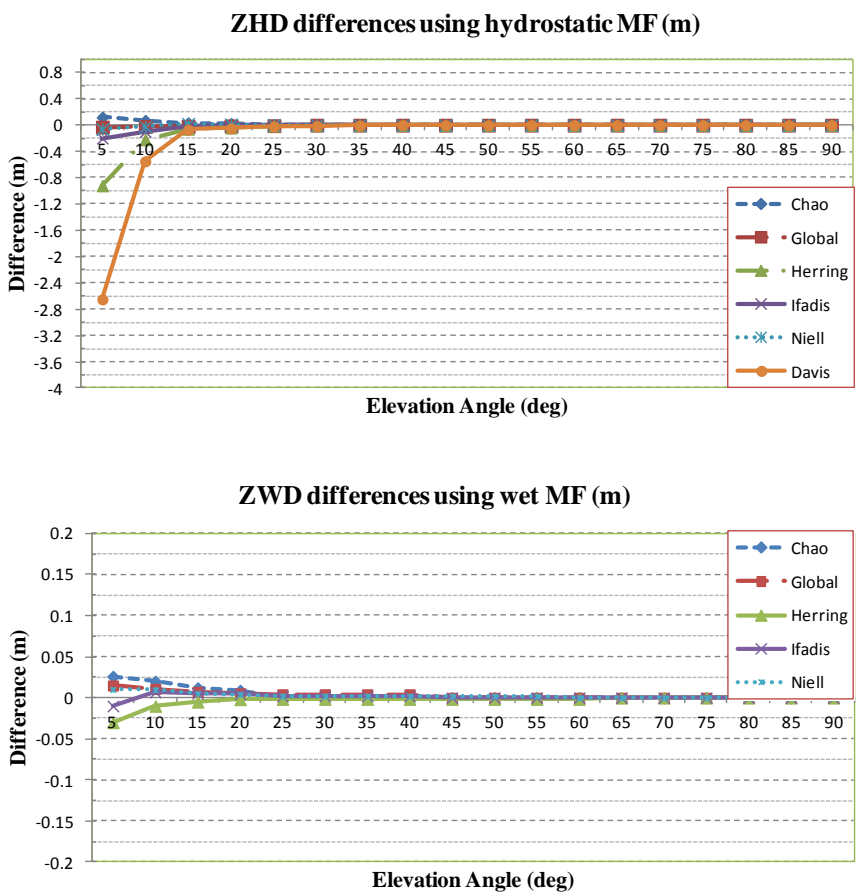

Figure 9: ZHD and ZWD differences between different MF and VMF1 for MDO1 (m)

The MFs Davis, Ifadis and Herring were limited in their accuracy ultimately by the dependence on surface temperature, which causes problems due to the variability in temperature in the boundary layer (from the surface up to 2000 $\mathrm{m}$ ) [23]. Even though the Ifadis MFs ares old, they still produce results similar to the newer models (Niell and Global) compared to VMF1. The GMFs performed equal to the VMF1 at the Equatorial station. This is due to the fact that the GMFs are a substitute model for the VMF1. Also the newer models such as GMFs and Niell perform better as they incorporate the latitude and height of the station, while the Davis and Ifadis models can operate with no station data, just using meteorological inputs. Consequently, the Ifadis, Niell and GMFs perform the best when compared to VMF1.

\section{Conclusions}

For the test data used, ZHD and ZTD comparison results show small discrepancies are present between different models and the IGS ZTD output. The Saastamoinen and Askne 
and Nordius models had the best performance, while the Baby models tend to overestimate the zenith delay. In general, in this analysis, all the tested models perform significantly better at the mid-latitudes than at the Equator. The ZWD models were not as easily distinguished as the ZHD. Due to the highly variable nature of the tropospheric water vapour, one or two parameters used in these models are often insufficient to describe the variations of the humidity with height. The error percentage of these models was high, which discourage the use of these models in practice.

For the mapping functions, the satellite signals that propagate at lower elevation angles experienced the largest delay. The Vienna Mapping Functions (VMF1) are regarded as the best for geodetic purposes. The analysis of different mapping functions show that the Niell, Global, Ifadis mapping functions perform the best when compared to VMF1. The differences among the examined mapping functions are smallest in value at the Northern Hemisphere station, and they are largest in value at the Equatorial station, just as the tropospheric delay models.

\section{REFERENCES}

[1] J. Askne, H. Nordius, Estimation of tropospheric delay for microwaves from surface weather data, Radio Science, $22-3$ (1987) 379-386.

[2] H. B. Baby, P. Gole, and J. Lavergnat, A model for the tropospheric excess path-length of radio-waves from surfac meteorlogical measuresments. Radio Science, 23-6 (1988) 1023-1038.

[3] A. L. Berman, The prediction of zenith range refraction from surface measurements of meteorological parameters. Jet Propulsion Laboratory, California Institute of Technology, Pasadena, Calif., 15 July, National Aeronautics and Space AdministrationTechnical Report 32-1602, (1976) 40 pp.

[4] J. Boehm, H. Schuh, Vienna mapping functions in VLBI analyses. Geophys. Res. Lett., 31 - 1 (2004) L01603.

[5] J., Boehm, A. Niell, P. Tregoning, and H. Schuh, Global Mapping Function (GMF): A new empirical mapping function based on numerical weather model data. Geophys. Res. Lett., 33-7 (2006A) L07304.

[6] J., Boehm, B. Werl, and H. Schuh, Troposphere mapping functions for GPS and very long baseline interferometry from European Centre for Medium-Range Weather Forecasts operational analysis data. J. Geophys. Res., 111-B2 (2006B) B02406.

[7] J. Boehm, J. Kouba, H. Schuh, Forecast Vienna Mapping Functions 1 for real-time analysis of space geodetic observations. Journal of Geodesy, 83- 5 (2009) 397-401.

[8] S. Byun, Y. Bar-Sever. A new type of troposphere zenith path delay product of the international GNSS service. Journal of Geodesy, 83 - 3 (2009) 1-7.

[9] C. C. Chao, A new method to predict wet aenith range correction from surface measurements. Jet Propulsion Laboratory, Pasadena, Calif., JPL Technical Report (1973) 32-1526.

[10] J. L. Davis, T. A. Herring, I. I. Shapiro, A. E. E. Rogers, G. Elgered, Geodesy by radio interferometry: Effects of atmospheric modeling errors on estimates of baseline length. Radio Sci., 20- 6 (1985) 1593-1607.

[11] J.D. Dodo, T.O. Idowu, Regional Assessment of the GPS Tropospheric Delay Models on the African GNSS Network, J. Of Emerging Trends in Engineering and Applied Sciences, 1- 1 (2010) 113-121. 
[12] J.M. Dow, R. E. Neilan, C. Rizos, The International GNSS Service in a changing landscape of Global Navigation Satellite Systems, Journal of Geodesy, 83 (2009) 191-198.

[13] A. El-Mowafy, Improving the Performance of RTK-GPS Reference Networks for Positioning in the Airborne Mode, Navigation, Journal of the Institute of Navigation (ION), 55- 3 (2008) 215-223.

[14] A. El-Mowafy, An Alternative Post-Processing Relative Positioning Approach Based on Precise Point Positioning, Journal of Surveying Engineering, 135- 2 (2009) 56-65.

[15] T. A. Herring, Modelling atmospheric delays in the analysis of space geodetic data, in Symposium on Refraction of Transatmospheric Signals in Geodesy, J. C. De Munk and T. A. Spoelstra, eds., Netherlands Geodetic Commission Series No. 36, 1992, pp. 157-164.

[16] H. Hopfield, Two-Quartic Tropospheric Refractivity Profile for Correcting Satellite Data. Journal of Geophysical Research Oceans, 74-18 (1969) 4487-4499.

[17] Ifadis, I.I., The atmospheric delay of radio waves: Modeling the elevation dependence on a global scale. Technical Report 38L. Goteborg, Sweden: Chalmers Univeristy of Technology (1986).

[18] Janes, H., R. Langley, and S. Newby, Analysis of tropospheric delay prediction models: comparisons with ray-tracing and implications for GPS relative positioning. Journal of Geodesy, Vol. 65, No. 3 (1991), pp.151-161.

[19] J. Kouba, Implementation and testing of the gridded Vienna Mapping Function 1 (VMF1). Journal of Geodesy, 82-4/5 (2008) 193-205.

[20] V.B. Mendes, R.B. Langley, Zenith tropospheric delay determination using prediction models: accuracy analysis, Cartografia e Cadastro, Instituto Português de Cartografia e Cadastro, 9 (1995) 41-47.

[21] V.B. Mendes, R.B. Langley, Tropospheric zenith delay prediction accuracy for highprecision GPS positioning and navigation. Navigation. 46-1 (1999) 25-34.

[22] V. B. Mendes, R. B. Langley, An analysis of high-accuracy tropospheric delay mapping functions. Physics and Chemistry of the Earth, Part A: Solid Earth and Geodesy, 25- 12 (2000) 809-812.

[23] A. E. Niell, Global mapping functions for the atmosphere delay at radio wavelengths. Journal of Geophysical Research-Solid Earth, 101(B2) (1996) 3227-3246.

[24] A. E. Niell, Improved atmospheric mapping functions for VLBI and GPS. Earth Planets and Space, 52-10 (2000) 699-702.

[25] J. Saastamoinen, Contributions to the theory of atmospheric refraction. Bulletin Géodésique, 107-1 (1973) 13-34.

[26] K. Saha, C. S. Raju, K. Parameswaran, A new hydrostatic mapping function for tropospheric delay estimation. J. of Atmospheric and Solar-Terrestrial Physics, 72-1 (2010) 125-134. 